\title{
Early versus Late Tracheostomy for Ventilator Dependence after Cardiovascular Surgery: Long-Term Results
}

\author{
Joseph M. Ladowski*, Hannah E. Downey, Bennet J. Ladowski, Joseph S. Ladowski \\ Lutheran Hospital of Indiana, Fort Wayne, USA \\ Email: ${ }^{*}$ jmladows@iupui.edu, hedowney@indiana.edu, ben.ladowski@gmail.com
}

Received June 11, 2013; revised July 11, 2013; accepted July 18, 2013

Copyright (C) 2013 Joseph M. Ladowski et al. This is an open access article distributed under the Creative Commons Attribution License, which permits unrestricted use, distribution, and reproduction in any medium, provided the original work is properly cited.

\begin{abstract}
Although concerns exist about the possibility of infecting a sternotomy wound by performing a tracheostomy early after cardiac surgery, it has been shown that tracheostomy performed before postoperative day 10 can improve outcomes for cardiovascular surgery patients who require long-term ventilation. We retrospectively reviewed all charts for patients who underwent cardiovascular surgery and required tracheostomy at a single-institution site from January 2005 to July 2012. Patients were divided into two groups based upon whether their tracheostomy was performed less than 10 days after their initial operation (early tracheostomy) or $\geq 10$ days after their initial operation (late tracheostomy). Preoperative demographics and postoperative complications were studied. There were no new mediastinitis episodes following tracheostomy. Early tracheostomy was associated with higher 90 -day ( $74.4 \%$ vs $56.8 \%$ ) and 180 -day (47.4\% vs $28.7 \%$, $\mathrm{p}<0.047)$ survival when compared to late tracheostomy. Early tracheostomy after cardiovascular surgery does not seem to be associated with an increased rate of mediastinitis but is associated with improved survival.
\end{abstract}

Keywords: Surgery Cardiovascular; Injury Lung; Intensive Care; Mediastinitis

\section{Introduction}

Long-term ventilator dependency following cardiovascular surgery occurs in up to $20 \%$ of all patients [1]. For patient comfort and to facilitate ease of mechanical ventilation, tracheostomy is commonly performed upon ventilator-dependent patients. The timing of tracheostomy is open to debate. Some investigators have indicated that patients who undergo earlier tracheostomy (less than ten days postoperatively) have decreased length of stay, morbidity and mortality [2]. Others have found that earlier tracheostomy in noncardiovascular surgery patients is associated with shorter times on mechanical ventilation and lower costs [3]. This study is a retrospective analysis of 137 consecutive patients who underwent cardiovascular surgery and required tracheostomy for long-term ventilator dependence. We analyzed these patients' records to determine whether early or late tracheostomy would affect development of sternotomy wound infections and whether a strategy of early sternotomy would allow for improved survival.

*Corresponding author.

\section{Materials and Methods}

We retrospectively reviewed the charts of 137 consecutive patients who underwent cardiovascular surgery and subsequent tracheostomy from January 2005 to July 2012 at Lutheran Hospital of Indiana. The types of initial operations are summarized in Table $\mathbf{1}$.

The time interval from initial operation to tracheostomy was decided clinically based upon the surgeon's

Table 1. Types of operations.

\begin{tabular}{cc}
\hline Operation & Number \\
\hline Coronary Artery Bypass & 88 \\
Abdominal Aortic Aneurysm (open) & 13 \\
Thoracic Aortic Aneurysm (open) & 9 \\
Single Valve Replacement or Repair & 15 \\
Double Valve Replacement or Repair & 11 \\
Combined Revascularization and Valve Replacement & 1 \\
Total & $\mathbf{1 3 7}$ \\
\hline
\end{tabular}


impression, aided by pulmonary consultation, that the patient would require prolonged ventilation. Tracheostomy was performed surgically with standard transverse neck incision and cephalocaudad tracheal incision followed by insertion of a tracheostomy appliance under direct vision. During this period, none of our tracheostomy procedures were done in a percutaneous fashion.

Statistical analysis of data was performed by subjecting survival data to Kaplan-Meier analysis. Significance was determined if $\mathrm{p}$ was $<0.05$ by Mantel-Cox log rank analysis.

Sixteen variables were studied for their univariate (Fisher's exact test for frequencies and analysis of variance for comparison of means) influence upon mortality. These variables are summarized in Table 2. All patient data was kept anonymous for reporting purposes.

\section{Results}

Outcomes for the 137 patients are summarized in Figure

1. Total mortality for the group was $42 \%$ at one year.

We began by analyzing the effect of earlier (less than ten days of initial operation) versus later (greater than or equal to ten days after initial operation) timing of tracheostomy. Thirty-three of the patients underwent tracheostomy within 10 days of their initial operation while 104 patients underwent tracheostomy greater than 10 days following their initial operation. Those patients who

Table 2. Variables studied for their possible association with mortality.

\begin{aligned} & \hline 1. Gender \\ & 2. Age \\ & 3. Redo surgery \\ & 4. Active smoker \\ & 5. History of alcohol abuse \\ & 6. Preoperative renal failure \\ & 7. Preoperative body mass index \\ & 8. Preoperative treatment for hypertension \\ & 9. Preoperative treatment for dyslipidemia \\ & 10. Preoperative treatment for diabetes mellitus \\ & 11. Postoperative sepsis \\ & 12. Postoperative renal failure requiring dialysis \\ & 13. Postoperative encephalopathy \\ & 14. Postoperative stroke \\ & 15. Postoperative myocardial infarction \\ & 16. Postoperative treatment for hyperglycemia \\ & \hline\end{aligned}

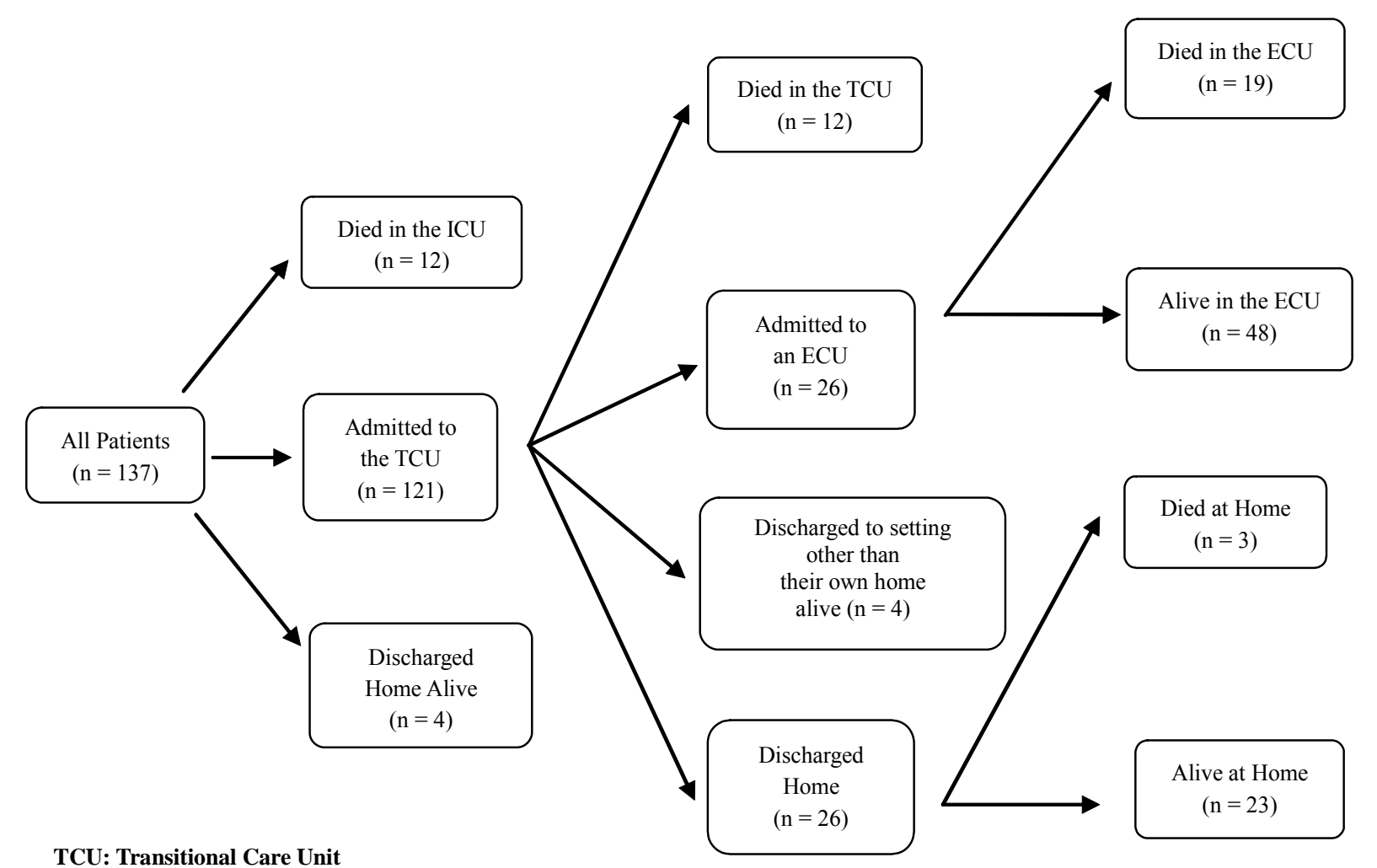

ECU: Extended Care Unit

Figure 1. Outcome for 137 patients who underwent tracheostomy after cardiovascular surgery. 
underwent tracheostomy within ten days of their initial procedure had 90- and 180-day survivals of $74.4 \%$ and $74.4 \%$ respectively while patients who underwent tracheostomy greater than ten days following their initial procedure had 90- and 180-day survivals of $56.8 \%$ and $28.7 \%$ respectively $(\mathrm{p}<0.047)$.

We next attempted to analyze whether or not the 33 patients who had early tracheostomy were similar in demographic makeup to the 104 patients who had late tracheostomy. The two groups were not significantly different in terms of gender, history of alcohol abuse, height, weight, body mass index (BMI), or preoperative presence of diabetes, hypertension, dyslipidemia, or renal failure. The two groups did differ regarding smoker status as $50 \%$ of the late tracheostomy group had never smoked compared to $39.4 \%$ of the early tracheostomy group ( $\mathrm{p}=$ $0.034)$. The late tracheostomy group was also older than the early tracheostomy group with $70.2 \%$ of the former being greater than 69 years while only $54.6 \%$ of the latter were older than $69(\mathrm{p}=0.012)$. Additionally we analyzed the type of initial surgical procedures that the patients had undergone. The early and late tracheostomy groups did not differ in type of initial operation (Table 3).

We then attempted to analyze whether the early versus late tracheostomy groups differed in incidences of postoperative complications. Table 4 summarizes these data. The late tracheostomy group had a lower incidence of postoperative stroke and a lower incidence of significant hyperglycemia. The incidence of dialysis-dependent renal failure, cardiac arrest, sepsis, myocardial infarction, and encephalopathy was not different between the two groups.
Importantly, the early and late tracheostomy groups who had undergone sternotomy for initial operation did not have a significant difference in incidence of developing sternal wound complications following their tracheostomy (none in each group).

\section{Discussion}

Patients who require tracheostomy for failure to wean from a ventilator after major cardiovascular surgery are known to have a markedly increased likelihood of mortality with hospital mortality as high as $49 \%$ [4]. It has been suggested that earlier tracheostomy might lead to better survival [2] for these patients.

Early tracheostomy after sternotomy has been found to be associated with increased risk of mediastinitis by one investigator [5]. This finding was not confirmed by subsequent studies [6-8]. We found no mediastinitis that developed after tracheostomy in the early or late group and were reassured that early tracheostomy does not necessarily increased the incidence of mediastinitis after sternotomy.

As expected, other investigations have found that patients who require tracheostomy after major cardiovascular surgery suffer increased mortality if they have significant preoperative renal failure or stroke morbidity present [9]. Similarly, patients who experience worsening organ function after cardiovascular surgery are at increased risk for death [10]. Although our late tracheostomy group was older, they had fewer smokers and experienced a lower incidence of postoperative stroke and a lower incidence of postoperative hyperglycemia. Despite

Table 3. Comparison of types of initial operations in early and late tracheostomy groups.

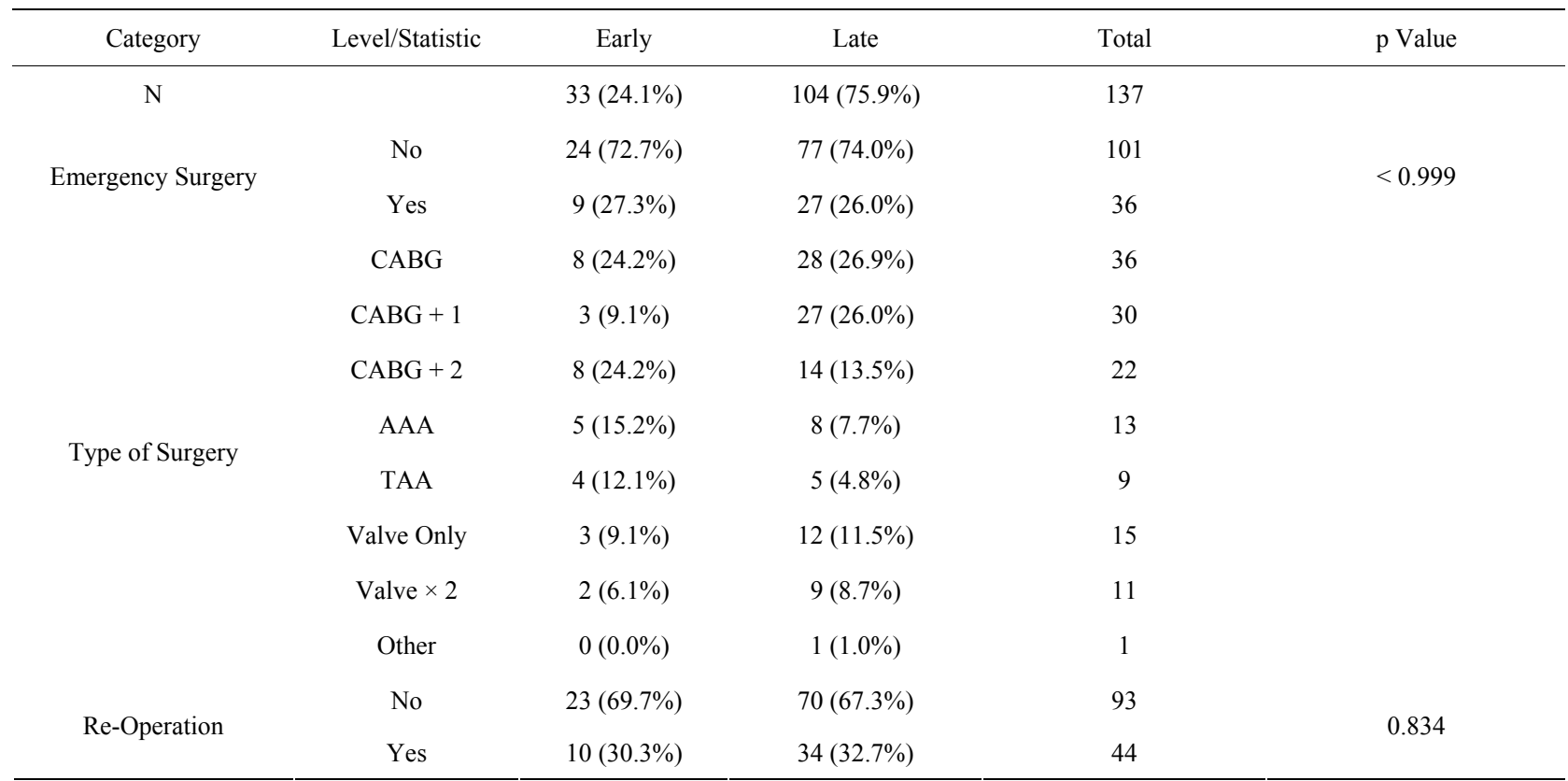


Table 4. Post-operative complications in early versus late tracheostomy groups.

\begin{tabular}{|c|c|c|c|c|c|}
\hline Category & Level/Stat & Early & Late & Total & $\mathrm{p}$ Value \\
\hline & $\mathrm{N}$ & $33(24.1 \%)$ & $104(75.9 \%)$ & 137 & \multirow{3}{*}{0.554} \\
\hline \multirow{2}{*}{ Renal Failure } & No & $17(51.5 \%)$ & $47(45.2 \%)$ & 64 & \\
\hline & Yes & $16(48.5 \%)$ & $57(54.8 \%)$ & 73 & \\
\hline \multirow{2}{*}{ Dialysis } & No & $27(81.8 \%)$ & $83(79.8 \%)$ & 110 & \\
\hline & Yes & $6(18.2 \%)$ & $21(20.2 \%)$ & 27 & \\
\hline \multirow{2}{*}{ Hyperglycemia } & No & $18(54.5 \%)$ & $78(75.0 \%)$ & 96 & \multirow{2}{*}{0.031} \\
\hline & Yes & $15(45.5 \%)$ & $26(25.0 \%)$ & 41 & \\
\hline \multirow{2}{*}{ Cardiac Arrest } & No & $30(90.9 \%)$ & $100(96.2 \%)$ & 130 & \multirow{2}{*}{0.359} \\
\hline & Yes & $3(9.1 \%)$ & $4(3.8 \%)$ & 7 & \\
\hline \multirow{2}{*}{ Sepsis } & No & $19(57.6 \%)$ & $60(57.7 \%)$ & 79 & \\
\hline & Yes & $14(42.4 \%)$ & $44(42.3 \%)$ & 58 & \\
\hline \multirow{2}{*}{ Myocardial Infarction } & No & $32(97.0 \%)$ & $103(99.0 \%)$ & 135 & \multirow{2}{*}{0.425} \\
\hline & Yes & $1(3.0 \%)$ & $1(1.0 \%)$ & 2 & \\
\hline Strol & No & $27(81.8 \%)$ & $100(96.2 \%)$ & 127 & \multirow{2}{*}{0.013} \\
\hline S110\% & Yes & $6(18.2 \%)$ & $4(3.8 \%)$ & 10 & \\
\hline \multirow{2}{*}{ Encephalopathy } & No & $28(84.8 \%)$ & $83(7.8 \%)$ & 111 & \multirow{2}{*}{0.617} \\
\hline & Yes & $5(15.2 \%)$ & $21(20.2 \%)$ & 26 & \\
\hline
\end{tabular}

a higher prevalence of these latter three risk factors, the early tracheostomy group was found to have improved survival compared to the late tracheostomy group.

Limitations of this study include the fact that this was a retrospective analysis with no attempt to randomize patients to early or later tracheostomy. None of the tracheostomies were performed with percutaneous techniques, which actually serves to provide standardization of technique throughout this study. Although the decision to perform early or later tracheostomy was made based upon subjective criteria of whether or not the patient could be weaned from mechanical ventilation, there does not seem to be a deliberate effort to perform later tracheostomy on the sicker patients, as evidenced by the lower incidence of postoperative complications (stroke and hyperglycemia) in the late tracheostomy patients.

\section{Conclusion}

Patients who require tracheostomy following cardiovascular surgery have significant risk of death with one year mortality of $42 \%$. A strategy of early tracheostomy, within ten days of the initial cardiovascular procedure, does not lead to an increase in risk of mediastinitis and seems to be associated with better chance for survival.

\section{Acknowledgements}

J. M. Ladowski-data analysis, data acquisition, manu- script.

H. E. Downey - data acquisition and analysis and manuscript preparation.

B. J. Ladowski-data acquisition and manuscript preparation.

J. S. Ladowski - original concept, data analysis, manuscript review, guarantor of paper.

\section{REFERENCES}

[1] S. C. Murthy, A. C. Arroliga, P. A. Walts, et al., "Ventilatory Dependence after Cardiovascular Surgery," Journal Thorac Cardiovasc Surgery, Vol. 134, No. 2, 2007, pp. 484-490. doi:10.1016/j.jtcvs.2007.03.006

[2] J. Devarajan, A. Vydyanathan, M. Xu, et al., "Early Tracheostomy Is Associated with Improved Outcome in Patients Who Require Prolonged Mechanical Ventilation after Cardiac Surgery," Journal of the American College of Surgeons, Vol. 214, No. 6, 2012, pp. 1008-1016. doi:10.1016/j.jamcollsurg.2012.03.005

[3] A. O. Brook, C. S. Sherman, J. Moley, et al., "Early Versus Late Tracheostomy in Patients Who Require Prolonged Mechanical Ventilation," American Journal of Critical Care, Vol. 9, No. 5, 2001, pp. 352-359.

[4] H. Ballota, T. Kandil, L. Generali, et al., "Tracheostomy after Cardiac Operation: In-Hospital and Long-Term Survival," The Annals of Thoracic Surgery, Vol. 92, No. 2, 2011, pp. 528-533. doi:10.1016/j.athoracsur.2011.02.002

[5] J. J. Curtis, N. C. Clark, C. A. McKenney, et al., "Tra- 
cheostomy: A Risk Factor for Mediastinitis after Cardiac Operations," The Annals of Thoracic Surgery, Vol. 72, No. 3, 2001, pp. 731-734. doi:10.1016/S0003-4975(01)02835-1

[6] S. A. Stamenkovic, I. S. Morgan, D. R. Pontefract and C. Campanella, "Is Early Tracheostomy Safe in Cardiac Patients with Median Sternotomy Incisions?" The Annals of Thoracic Surgery, Vol. 69, No. 4, 2000, pp. 1152-1154.

[7] P. B. Rahmanian, D. H. Adams, J. G. Castillo, et al., "Tracheostomy Is Not a Risk Factor for Deep Sternal Wound Infections after Cardiac Surgery," The Annals of Thoracic Surgery, Vol. 84, No. 6, 2007, pp. 1984-1991. doi:10.1016/j.athoracsur.2007.07.024

[8] S. D. Force, D. L. Miller, R. Petersen, et al., "Incidence of Deep Sternal Wound Infections after Tracheostomy in
Cardiac Surgery Patients," The Annals of Thoracic Surgery, Vol. 80, No. 2, 2005, pp. 618-621. doi:10.1016/j.athoracsur.2005.02.056

[9] M. Engoran, N. F. Buderer and A. Zacharias, "Long Term Survival and Health Status after Prolonged Mechanical Ventilation after Cardiac Surgery," Critical Care Medicine, Vol. 28, No. 8, 2000, pp. 2742-2749. doi:10.1097/00003246-200008000-00010

[10] P. A. Walts, S. C. Murthy, A. C. Arroliga, et al., "Tracheostomy after Cardiovascular Surgery: An Assessment of Long-Term Outcome," The Journal of Thoracic and Cardiovascular Surgery, Vol. 131, No. 4, 2006, pp. 830837. doi:10.1016/j.jtcvs.2005.09.038 